\title{
La educación en tiempos de incertidumbre: un esfuerzo común de entendimiento
}

\author{
Marta García-Lastra y Maddalena Colombo'
}

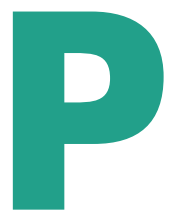

resentamos en este nuevo número de la Revista de Sociología de la Educación algunos de los trabajos compartidos en el congreso organizado por la Associazione Italiana di Sociologia (AIS) y en el encuentro del Comité de Investigación de Sociología de la Educación de la Federación Española de Sociología (FES), celebrados ambos en 2021.

Dos encuentros atravesados por la COVID que ha imposibilitado vernos de manera presencial en cada uno de ellos, pero que aun así han puesto de manifiesto el interés de la Sociología de la Educación del Sur de Europa por buscar lugares de encuentro y de compromiso mutuo.

En mayo de 2021 se celebró desde Milán el 1. ${ }^{\circ}$ Congreso Internacional «Sociology of Education in Southern Europe a step foward (SESE)». Pospuesto desde mayo de 2020 por la pandemia, investigadores e investigadoras junior y senior fueron convocados a participar en él. Se celebró de forma telemática y supuso un indicador más de las fructíferas relaciones que desde hace tiempo se mantienen entre las diferentes asociaciones de Sociología de la Educación del sur del continente: Associaz̧ione Italiana di Sociologia (Sezione di Sociologia dell'Educazione), Association Française de Sociologie (Réseau Thématique 'Sociologie de l'Education et de la Formation'), Associação Portuguesa de Sociologia (Seção de 'Sociologia da Educação') y Federación Española de Sociología (Comité de Investigación de 'Sociología de la Educación'), y que se han materializado en otros encuentros previos, desde la ya lejana conferencia que celebramos en Lisboa en 2015 o la de Córdoba de 2017.

También la pandemia nos obligó a cancelar el congreso de la Federación Española de Sociología que iba a celebrarse en Murcia en 2021. En este congreso, como comité de investigación, nos encontrábamos habitualmente para compartir no solo conocimientos, investigaciones, sino también para seguir tejiendo esa red que comenzó en la década de los noventa como relata uno de los artículos de este número. Con el título Educación post-covid: retos para la sociedad, nos reunimos también de manera telemática en el mes de octubre de 2021 para dialogar, entre otros temas, sobre la realidad de la sociedad postpandemia y sus consecuencias en el mundo de la educación.

Al mismo tiempo, la sección Educación de la AIS se reunió en septiembre de 2021 en Cagliari para discutir acerca de esta misma cuestión, Una comunidad reflexiva: desafío, misión y perspectivas para la sociología de la educación, analizando todos los cambios provocados por la crisis económica y la lucha contra el virus.

1 Marta García Lastra, Universidad de Cantabria, marta.garcia@unican.es y Maddalena Colombo, Università Cattolica del Sacro Cuore (Milán), maddalena.colombo@unicatt.it. 
Estos encuentros, y los trabajos en ellos presentados entre los que se encuentran algunos de los seleccionados y publicados en este número de RASE, ponen de manifiesto el interés por aunar esfuerzos en el trabajo de la Sociología de la Educación en contextos sociales más o menos similares y con denominadores comunes.

La pandemia ha significado una enorme «prueba de estrés» para todos los sistemas sociales, por lo que el contenido y la metodología de nuestra investigación no pueden evitar considerarla como una «línea divisoria» entre el antes y el después. Se han mantenido temas habituales en nuestra disciplina, pero también han ido apareciendo otras sensibilidades en las que la pandemia, sus efectos sobre la escuela, los sistemas educativos y las prácticas pedagógicas han estado presentes como no podía ser de otra manera, dado el interés de la Sociología de la Educación por las consecuencias de esta pandemia, también social.

¿Por qué los sociólogos y sociólogas de Italia, España, Francia y Portugal pretenden cultivar las bases teóricas y empíricas de una Sociología de la Educación desde una perspectiva del Sur de Europa?

Destacamos el «Sun» no sólo como una referencia geográfica sino también como una referencia cultural y simbólica; cada uno en su vida puede sentirse del «Sur» en comparación con otras personas, puede ser marcado como «del Sur» con significados tanto negativos como positivos, asociados a estar lejos, a lo marginal, a lo exótico, de cualquier manera, diferente de lo que se considera centro.

El «Sur» es principalmente un sentimiento y una experiencia, pero sabemos que está estructurado a través de los antiguos procesos de categorización, estratificación y nominación. En cierto sentido, «Sun» señala las diferencias sociales y culturales, sus raíces históricas y su ambivalencia actual: según la situación, «Sur» es una metáfora del sufrimiento humano, la desventaja, la exclusión, la explotación, la desigualdad, la pobreza, la periferia; pero también podría significar la resistencia a ese sufrimiento humano, los lazos comunitarios, el localismo, la solidaridad, la resiliencia y la creatividad.

La exhaustividad (y también la ambivalencia) de la categoría «Sur» es visible al leer los siete artículos de este número que abordan algunas de las principales preocupaciones sobre los procesos educativos en Europa.

El abanico de temas abarca tanto cuestiones generales, como las desigualdades y las políticas educativas nacionales, como cuestiones específicas, como la educación de adultos, la comunidad local de aprendizaje y los servicios de atención a la infancia. Además de estos trabajos (basados tanto en la evidencia como en la literatura), merece la pena que el lector no español conozca el desarrollo de una Sociología «internacional» de la educación en España en las últimas décadas, como se narra en uno de ellos.

En el primer artículo, Orazio Giancola y Luca Salmieri (Universidad de Roma «La Sapienza») ponen en discusión la existencia de un régimen de bienestar en el sur de Europa a la luz de las diferentes especificidades nacionales. Sostienen que las comparaciones entre países de los sistemas y resultados educativos no se han centrado lo suficiente como para proporcionar un marco sólido que permita identificar un patrón educativo común o diferente entre países como Grecia, Italia, Malta, Portugal y España. Por lo tanto, su objetivo es comprender si existe un espacio educativo sureuropeo en relación con las características estructurales internas y las desigualdades educativas, y si los sistemas educativos nacionales individuales tienden al isomorfismo, al polimorfismo o a la hibridación.

El segundo artículo, redactado por Xavier Pons (Université de Paris-Est Créteil), presenta las primeras conclusiones de una revisión bibliográfica sobre la forma en que los estudiosos que trabajan sobre 
las políticas educativas francesas, italianas y portuguesas consideran, y posiblemente conceptualizan, los contextos de las políticas. A través de 175 artículos, muestra que los autores tienden a contextualizar poco el análisis de las políticas educativas y que no existen operaciones de contextualización específicas para los autores que trabajan sobre los países del sur de Europa. Las numerosas limitaciones metodológicas sugieren la necesidad de una problematización específica, en particular en torno a las lógicas de vernacularización de los enunciados y requisitos de las políticas globales.

La tercera contribución, Vanessa Pinto Carvalho da Silva (Centro de Investigaçao e Estudos de Sociologia), realiza un análisis comparativo de la educación de personas adultas en Portugal, Grecia, Italia y España, compartiendo similitudes y diferencias que se encuentran en los sistemas de educación y formación de adultos. Mediante el uso de la revisión de la literatura y el análisis secundario de los datos de la Encuesta de Educación de Adultos (2016), la autora describe la relación entre la participación en el aprendizaje permanente en cada uno de estos países, las principales barreras y los resultados.

En el cuarto artículo escrito por Rafael Feito Alonso (Universidad de Madrid), Leopoldo Cabrera Rodríguez (Universidad de La Laguna) y José Beltrán Llavador (Universidad de Valencia), se realiza un breve repaso histórico por la Sociología de la Educación en España. Desde 1990 el grupo ha crecido en número y riqueza de estudios, al tiempo que su apertura internacional queda atestiguada desde 2015 con la organización de múltiples seminarios y conferencias con invitados extranjeros. Actualmente la Sociología de la Educación española cuenta con dos revistas de impacto, RASE (creada en 2008) y RISE (en 2012).

El quinto artículo lo firman Silvia Carrasco y Marina Pibernat, investigadoras de EMIGRA-CER Migracions de la Universitat Autònoma de Barcelona, en el que presentan un trabajo basado en algunos resultados de un estudio prospectivo a partir de entrevistas con profesorado de centros de secundaria de Madrid y Barcelona en el que tratan el impacto de la pandemia y el cierre de la escuela en alumnado desde un enfoque comparativo de origen nacional y género.

El estudio realizado por Francesca Buriel (Universidad Autónoma de Barcelona), sexto artículo de este número, trata de una comunidad local de aprendizaje transformada en comunidad digital debido al cierre de la escuela. La autora examina diferentes componentes y dinámicas de la comunicación familia-escuela en ocho escuelas primarias de Cataluña (España). Mediante un proceso multimodal de recogida de datos para analizar documentos escolares, cuestionarios digitales y entrevistas en línea a grupos mixtos (triangulación de datos), el trabajo proporciona información útil sobre cómo el alumnado ha sido habitualmente excluido de la toma de decisiones y de la transformación educativa, y reivindica su presencia para mejorar la educación participativa.

El último artículo escrito por Manuel Ángel Río Ruiz, Rubén Martín Gimeno y María Ortega Gómez (Universidad de Sevilla y Universidad Pablo de Olavide) aborda una tendencia reciente en los servicios de atención a la infancia en Andalucía. El análisis muestra la expansión de la escolarización en el ciclo 0-3 sustentada por la proliferación de centros infantiles privados concertados. También identifica diferentes tendencias de exclusión de este sistema, las que se producen por motivos económicos (familias que se sitúan por debajo del umbral de la pobreza) y por autoexclusión (familias de las clases bajas que se autoexcluyen o retrasan al máximo la escolarización por falta de subvenciones e incentivos económicos).

Completa este número una reseña elaborada el profesor Juan Sebastián Fernández-Prados del libro de la profesora de la Universidad de Almería Teresa García Gómez titulado Palabras y pedagogía desde Paulo 
Freire, figura referente para el campo de la educación de quien se ha recordado en 2021 el centenario de su nacimiento.

Deseamos que esta colección de artículos sea útil para los sociólogos y sociólogas de la educación, evaluable también como un indicador de una unión científica positiva entre expertos y ciudadanía del «sur de Europa», que se ampliará en los próximos años gracias a la contribución de investigadores e investigadoras individuales y, sobre todo, de las organizaciones colectivas a las que pertenecen. 